\title{
RECENT DEVELOPMENT OF SUPERSYMMETRIC GAUGE THEORIES ON LATTICE
}

\author{
FUMIHIKO SUGINO \\ Okayama Institute for Quantum Physics, Kyoyama 1-9-1 \\ Okayama, 700-0015, Japan \\ fumihiko_sugino@pref.okayama.lg.jp
}

\begin{abstract}
First, we give a brief review of recent development of lattice formulations for supersymmetric Yang-Mills (SYM) theories with extended supersymmetry, which preserves a part of supersymmetry on lattice. For cases of two dimensions, we can see that lattice models in such formulations lead to the target continuum theories with no fine-tuning. Namely, supersymmetries or some other symmetries not realized on the lattice are automatically restored in the continuum limit.

Next, we consider a mass deformation to $\mathcal{N}=(8,8) \mathrm{SYM}$ and present its lattice formulation with keeping two supercharges. It provides a nonperturbative framework to investigate IIA matrix string theory. Moreover, since it has fuzzy sphere solutions around which four-dimensional theory is deconstructed, it will serve a nonperturbative formulation of four-dimensional $\mathcal{N}=4 \mathrm{SYM}$ which requires no fine-tuning. The rank of the gauge group is not restricted to large $N$. It opens a quite interesting possibility to test AdS/CFT correspondence in a stringy regime where string loop effects cannot be neglected. Also, for two-dimensional $\mathcal{N}=(4,4) \mathrm{SYM}$, a similar argument is possible to obtain four-dimensional $\mathcal{N}=2 \mathrm{SYM}$ on noncommutative space.
\end{abstract}

Keywords: Supersymmetric gauge theory; lattice gauge theory; matrix models.

PACS Numbers: 11.15.Ha, 11.30.Pb, 02.10.Yn

\section{Introduction}

First, we start with a brief review of recent development of lattice formulations of supersymmetric gauge theories. Lattice formulations mean to reformulate quantum field theories on discretized space-time, which is not only a ultra-violet (UV) regularization but more importantly gives an nonperturbative construction of the theories. Also, they makes possible computer simulations which serve a conventional tool to investigate nonperturbative aspects of quantum field theories. It is quite interesting to extend such formulations for supersymmetric gauge field theories, since they have been an intriguing candidate describing physics beyond the standard model and they have been found to have a close connection to string theory via matrix string conjectures ${ }^{1-3}$ and AdS/CFT correspondence. ${ }^{4}$ 
However, there is a notorious difficulty regarding a realization of supersymmetry on lattice. Typical supersymmetry algebra

$$
\left\{Q_{\alpha}, Q_{\beta}\right\} \sim \gamma_{\alpha \beta}^{\mu} p_{\mu}
$$

contains momenta $p_{\mu}$ which act as derivatives to continuous space-time. Naively, derivative operators are replaced with difference operators upon discretizing spacetime to lattice. Note that the Leibniz rule, which holds for derivative operators, becomes broken for difference operators by the quantity $\mathcal{O}(a)$. Here, $a$ is a lattice spacing. It generally leads to an explicit breaking of supersymmetry in a lattice action by the amount $\mathcal{O}(a)$. So, near the continuum limit, i.e. for $a$ being small, the lattice action $S^{\text {lat }}$ can be written as the sum of the corresponding continuum action $S^{\text {cont }}$ and the $\mathcal{O}(a)$ quantity $\tilde{S}$ :

$$
S^{\text {lat }}=S^{\mathrm{cont}}+\tilde{S}
$$

As long as at the classical level, $\tilde{S}$ is irrelevant in the continuum limit $(a \rightarrow 0)$. But, quantum mechanically, terms in $\widetilde{S}$ may receive radiative corrections which become diverge as $a \rightarrow 0$. Note that $a$ plays the role of a UV cutoff. Suppose a radiative correction from some term in $\tilde{S}$ behaves as $\mathcal{O}\left(\frac{1}{a^{\sharp}}\right)$. Then, the term becomes relevant when

$$
\mathcal{O}(a) \times \mathcal{O}\left(\frac{1}{a^{\sharp}}\right)=\mathcal{O}\left(\frac{1}{a^{\sharp}-1}\right) \geq \mathcal{O}\left(a^{0}\right) .
$$

It means that supersymmetry breaking terms in $\tilde{S}$ can be relevant at the quantum level, and then we cannot reach the desired supersymmetric theory by sending $a \rightarrow 0$ naively in (2). In that case, in order to obtain the supersymmetric continuum theory, we should remove such supersymmetry breaking relevant operators in advance. This procedure is called as fine-tuning.

Since it is quite cumbersome to carry out fine-tuning in actual computer simulations, lattice models requiring no fine-tuning are practically best (although a few operators to be fine-tuned are perhaps acceptable). So, let us focus on cases that lattice actions have "good symmetries" which protect from generating symmetrybreaking radiative corrections. For some (lower-dimensional) gauge theories with extended supersymmetry, it has been found that not all supersymmetries, but some part of "nilpotent" supersymmetry not generating translations plays a role of the "good symmetries". Some of such examples are ${ }^{a}$

- $\mathcal{N}=(2,2)$ supersymmetric Yang-Mills (SYM) theory in two dimensions ${ }^{7-9}$

- $\mathcal{N}=(4,4) \mathrm{SYM}$ in two dimensions ${ }^{8,10}$

- $\mathcal{N}=(8,8)$ SYM in two dimensions ${ }^{11}$

- $\mathcal{N}=(2,2)$ supersymmetric QCD (SQCD) in two dimensions. ${ }^{12-16}$

${ }^{\text {a }}$ For recent reviews, see Refs. 5 and 6. 
There is a perturbative argument for these models to restore full supersymmetry in the continuum limit. Moreover, nonperturbative check of the restoration has been done by computer simulation for two-dimensional $\mathcal{N}=(2,2)$ SYM with the gauge group $G=S U(2)$ in Ref. 17 and $G=S U(N)(N=2,3,4,5)$ in Ref. 18 .

The construction of the theories in Refs. 7, 10, 11, 12, 13 is based on deconstruction from zero-dimensional matrix models, which is possible for the gauge group $G=U(N)$ but not for $S U(N)$. On the other hand, the construction in Refs. 8, 9, 14,15 is quite analogous to the conventional lattice gauge theory, and possible for both of the gauge groups $G=U(N)$ and $S U(N)$. Ref. 19 has shown that the model constructed in Ref. 7 is free from the sign problem and gives the same physics as that in Ref. 8 after an appropriate treatment of the overall $U(1)$ modes.

In what follows, we present a new lattice formulation for two-dimensional $\mathcal{N}=$ $(8,8)$ SYM theory with the gauge groups $G=U(N), S U(N)$ based on Ref. 20. By using a plane-wave like mass deformation, flat directions of scalar fields are lifted with preserving two "nilpotent" supercharges. Next, we discuss a scenario to obtain four-dimensional $\mathcal{N}=4$ SYM from a fuzzy $S^{2}$ background of the twodimensional theory with $G=U(N) \cdot{ }^{20,21}$ Note that the gauge group of the fourdimensional theory is $U(k)$ with $k$ being arbitrary. As related work, Ref. 22 leads to three-dimensional $\mathcal{N}=8 \mathrm{SYM}$ from a fuzzy $S^{2}$ background in the plane-wave matrix model which is a matrix quantum mechanics. ${ }^{23}$ Also, Ref. 24 obtains fourdimensional $\mathcal{N}=4 \mathrm{SYM}$ on $\mathbf{R} \times S^{3}$ with the gauge group $U(\infty)$, i.e. a planar gauge theory, from the plane-wave matrix model. Ref. 25 gives a perturbative argument about parameters to be fine-tuned in a lattice formulation for four-dimensional $\mathcal{N}=4$ SYM preserving one supercharge. The similar construction but starting from a mass-deformed version of the lattice formulation for two-dimensional $\mathcal{N}=(8,8)$ SYM in Ref. 11 is discussed in Ref. 26.

\section{Continuum Two-Dimensional $\mathcal{N}=(8,8) \mathrm{SYM}$}

Let us start with the Euclidean action of $\mathcal{N}=(8,8)$ SYM in continuum twodimensional space-time with the gauge group $G=U(N)$ or $S U(N)$ :

$$
\begin{aligned}
S_{0}= & \frac{2}{g_{2 d}^{2}} \int d^{2} x \operatorname{tr}\left[\frac{1}{2} F_{12}^{2}+\frac{1}{2}\left(D_{\mu} X^{I}\right)^{2}-\frac{1}{4}\left[X^{I}, X^{J}\right]^{2}\right. \\
& \left.+\frac{1}{2} \Psi^{T}\left(D_{1}+\gamma_{2} D_{2}\right) \Psi+\frac{i}{2} \Psi^{T} \gamma_{I}\left[X^{I}, \Psi\right]\right],
\end{aligned}
$$

where $\mu=1,2$, and $I, J=3,4, \ldots, 10 . F_{12}=\partial_{1} A_{2}-\partial_{2} A_{1}+i\left[A_{1}, A_{2}\right]$ is a gauge field strength, and $D_{\mu}=\partial_{\mu}+i\left[A_{\mu}, \cdot\right]$ gauge covariant derivatives. $X^{I}(I=3,4, \ldots, 10)$ represent eight adjoint scalars, and $\Psi$ a sixteen-component adjoint fermion. $\gamma_{I^{\prime}}$ $\left(I^{\prime}=2,3, \ldots, 10\right)$ are imaginary symmetric $16 \times 16$ matrices satisfying $\left\{\gamma_{I^{\prime}}, \gamma_{J^{\prime}}\right\}=$ $-2 i \delta_{I^{\prime} J^{\prime}} \mathbb{1}_{16}$.

It is convenient to rewrite (4) in the language of topological twist as the form of balanced topological field theory. ${ }^{27}$ The eight scalars $X^{I}$ and the sixteen components 
of $\Psi$ are denoted as

$$
\begin{aligned}
X^{I} \Rightarrow & \begin{cases}X_{i} & (i=3,4) \\
B_{\mathrm{A}} & (\mathrm{A}=1,2,3) \\
C=2 X_{8}, \phi_{ \pm}=X_{9} \pm i X_{10}\end{cases} \\
\Psi & \Rightarrow\left\{\begin{array}{l}
\psi_{+\mu}, \rho_{+i}, \chi_{+\mathrm{A}}, \eta_{+} \\
\psi_{-\mu}, \rho_{-i}, \chi_{-\mathrm{A}}, \eta_{-} .
\end{array}\right.
\end{aligned}
$$

The precise form of the field redefinition is given in Appendix A with the explicit form of the gamma matrices. There are appropriate two supercharges $Q_{+}^{(0)}, Q_{-}^{(0)}$ to recast the action to the $Q_{+}^{(0)} Q_{-}^{(0)}$ exact form: ${ }^{\text {b }}$

$$
\begin{aligned}
S_{0}= & Q_{+}^{(0)} Q_{-}^{(0)} \mathcal{F}_{0} \\
\mathcal{F}_{0}= & \frac{1}{g_{2 d}^{2}} \int d^{2} x \operatorname{tr}\left[-i B_{\mathrm{A}} \Phi_{\mathrm{A}}-\frac{1}{3} \epsilon_{\mathrm{ABC}} B_{\mathrm{A}}\left[B_{\mathrm{B}}, B_{\mathrm{C}}\right]\right. \\
& \left.-\psi_{+\mu} \psi_{-\mu}-\rho_{+i} \rho_{-i}-\chi_{+\mathrm{A}} \chi_{-\mathrm{A}}-\frac{1}{4} \eta_{+} \eta_{-}\right]
\end{aligned}
$$

with

$$
\begin{aligned}
& \Phi_{1}=2\left(-D_{1} X_{3}-D_{2} X_{4}\right), \quad \Phi_{2}=2\left(-D_{1} X_{4}+D_{2} X_{3}\right), \\
& \Phi_{3}=2\left(-F_{12}+i\left[X_{3}, X_{4}\right]\right) .
\end{aligned}
$$

The supercharges $Q_{ \pm}^{(0)}$ act as

$$
\begin{aligned}
& Q_{ \pm}^{(0)} A_{\mu}=\psi_{ \pm \mu}, \quad Q_{ \pm}^{(0)} \psi_{ \pm \mu}= \pm i D_{\mu} \phi_{ \pm}, \quad Q_{\mp}^{(0)} \psi_{ \pm \mu}=\frac{i}{2} D_{\mu} C \mp \tilde{H}_{\mu} \\
& Q_{ \pm}^{(0)} \tilde{H}_{\mu}=\left[\phi_{ \pm}, \psi_{\mp \mu}\right] \mp \frac{1}{2}\left[C, \psi_{ \pm \mu}\right] \mp \frac{i}{2} D_{\mu} \eta_{ \pm}
\end{aligned}
$$

to gauge multiplets $\left(A_{\mu}, \psi_{ \pm \mu}, \tilde{H}_{\mu}\right)$ with $\tilde{H}_{\mu}$ auxiliary fields, as

$$
\begin{aligned}
& Q_{ \pm}^{(0)} X_{i}=\rho_{ \pm i}, \quad Q_{ \pm}^{(0)} \rho_{ \pm i}=\mp\left[X_{i}, \phi_{ \pm}\right], \quad Q_{\mp}^{(0)} \rho_{ \pm i}=-\frac{1}{2}\left[X_{i}, C\right] \mp \tilde{h}_{i}, \\
& Q_{ \pm}^{(0)} \tilde{h}_{i}=\left[\phi_{ \pm}, \rho_{ \pm i}\right] \mp \frac{1}{2}\left[C, \rho_{ \pm i}\right] \pm \frac{1}{2}\left[X_{i}, \eta_{ \pm}\right]
\end{aligned}
$$

to $X_{i}$ multiplets $\left(X_{i}, \rho_{ \pm i}, \tilde{h}_{i}\right)$ with $\tilde{h}_{i}$ auxiliary fields, as

$$
\begin{aligned}
& Q_{ \pm}^{(0)} B_{\mathrm{A}}=\chi_{ \pm \mathrm{A}}, \quad Q_{ \pm}^{(0)} \chi_{ \pm \mathrm{A}}= \pm\left[\phi_{ \pm}, B_{\mathrm{A}}\right], \quad Q_{\mp}^{(0)} \chi_{ \pm \mathrm{A}}=\frac{1}{2}\left[C, B_{\mathrm{A}}\right] \mp H_{\mathrm{A}}, \\
& Q_{ \pm}^{(0)} H_{\mathrm{A}}=\left[\phi_{ \pm}, \chi_{\mp \mathrm{A}}\right] \pm \frac{1}{2}\left[B_{\mathrm{A}}, \eta_{ \pm}\right] \mp \frac{1}{2}\left[C, \chi_{ \pm \mathrm{A}}\right]
\end{aligned}
$$

to $B_{\mathrm{A}}$ multiplets $\left(B_{\mathrm{A}}, \chi_{ \pm \mathrm{A}}, H_{\mathrm{A}}\right)$ with $H_{\mathrm{A}}$ auxiliary fields, and as

$$
\begin{array}{ll}
Q_{ \pm}^{(0)} C=\eta_{ \pm}, & Q_{ \pm}^{(0)} \eta_{ \pm}= \pm\left[\phi_{ \pm}, C\right], \quad Q_{\mp}^{(0)} \eta_{ \pm}=\mp\left[\phi_{+}, \phi_{-}\right], \\
Q_{ \pm}^{(0)} \phi_{ \pm}=0, & Q_{\mp}^{(0)} \phi_{ \pm}=\mp \eta_{ \pm}
\end{array}
$$

to parameter multiplets $\left(C, \phi_{ \pm}, \eta_{ \pm}\right)$.

${ }^{\mathrm{b}}$ This is essentially obtained by dimensional reduction from Eq. (4.12) in Ref. 28. 
From the above transformation property, we find that $Q_{ \pm}^{(0)}$ are "nilpotent" in the sense that

$$
\begin{aligned}
\left(Q_{+}^{(0)}\right)^{2} & =\left(\text { infinitesimal gauge transformation by } \phi_{+}\right), \\
\left(Q_{-}^{(0)}\right)^{2} & =\left(\text { infinitesimal gauge transformation by }-\phi_{-}\right), \\
\left\{Q_{+}^{(0)}, Q_{-}^{(0)}\right\} & =(\text { infinitesimal gauge transformation by } C) .
\end{aligned}
$$

Thus, $S_{0}$ in (8) is manifestly invariant under $Q_{ \pm}^{(0)}$ supersymmetries. Note that $\mathcal{F}_{0}$ is gauge invariant. There is an $S U(2)_{R}$ symmetry as another manifest symmetry of $S_{0}$, which is a subgroup of $S O(8)$ internal symmetry of the theory. Its generators $J_{++}, J_{--}$and $J_{0}$ are expressed as

$$
\begin{gathered}
J_{ \pm \pm}=\int d^{2} x\left[\psi_{ \pm \mu}^{\alpha}(x) \frac{\delta}{\delta \psi_{\mp \mu}^{\alpha}(x)}+\chi_{ \pm \mathrm{A}}^{\alpha}(x) \frac{\delta}{\delta \chi_{\mp \mathrm{A}}^{\alpha}(x)}-\eta_{ \pm}^{\alpha}(x) \frac{\delta}{\delta \eta_{\mp}^{\alpha}(x)}\right. \\
\left. \pm 2 \phi_{ \pm}^{\alpha}(x) \frac{\delta}{\delta C^{\alpha}(x)} \mp C^{\alpha}(x) \frac{\delta}{\delta \phi_{\mp}^{\alpha}(x)}\right] \\
J_{0}=\int d^{2} x\left[\psi_{+\mu}^{\alpha}(x) \frac{\delta}{\delta \psi_{+\mu}^{\alpha}(x)}-\psi_{-\mu}^{\alpha}(x) \frac{\delta}{\delta \psi_{-\mu}^{\alpha}(x)}+\chi_{+\mathrm{A}}^{\alpha}(x) \frac{\delta}{\delta \chi_{+\mathrm{A}}^{\alpha}(x)}\right. \\
-\chi_{-\mathrm{A}}^{\alpha}(x) \frac{\delta}{\delta \chi_{-\mathrm{A}}^{\alpha}(x)}+\eta_{+}^{\alpha}(x) \frac{\delta}{\delta \eta_{+}^{\alpha}(x)}-\eta_{-}^{\alpha}(x) \frac{\delta}{\delta \eta_{-}^{\alpha}(x)} \\
\left.+2 \phi_{+}^{\alpha}(x) \frac{\delta}{\delta \phi_{+}^{\alpha}(x)}-2 \phi_{-}^{\alpha}(x) \frac{\delta}{\delta \phi_{-}^{\alpha}(x)}\right]
\end{gathered}
$$

with $\alpha=1, \ldots, \operatorname{dim}(G)$ labeling a basis of gauge group generators, and satisfy

$$
\left[J_{0}, J_{ \pm \pm}\right]= \pm 2 J_{ \pm \pm}, \quad\left[J_{++}, J_{--}\right]=J_{0} .
$$

$J_{0}$ is a generator of $U(1)_{R}$ rotation, which is contained in $S U(2)_{R}$ as its Cartan subalgebra. Under the $S U(2)_{R}$ rotation, each of $\left(\psi_{+\mu}, \psi_{-\mu}\right),\left(\chi_{+\mathrm{A}}, \chi_{-\mathrm{A}}\right)$, and $\left(\eta_{+},-\eta_{-}\right)$ transforms as a doublet, and $\left(\psi_{+}, C,-\phi_{-}\right)$as a triplet. Note that a pair of the supercharges $\left(Q_{+}^{(0)}, Q_{-}^{(0)}\right)$ also forms a doublet. In particular,

$$
\left[J_{ \pm \pm}, Q_{ \pm}^{(0)}\right]=0, \quad\left[J_{ \pm \pm}, Q_{\mp}^{(0)}\right]=Q_{ \pm}^{(0)} .
$$

Fermions with the suffix \pm carry the $J_{0}$-charge \pm 1 , and $\phi_{ \pm}$carry \pm 2 . Since $\mathcal{F}_{0}$ is invariant under the $S U(2)_{R}$, so is

$$
S_{0}=Q_{+}^{(0)} Q_{-}^{(0)} \mathcal{F}_{0}=\epsilon_{\gamma \delta} Q_{\gamma}^{(0)} Q_{\delta}^{(0)} \mathcal{F}_{0} \quad(\gamma, \delta= \pm)
$$

\section{Mass Deformation of Two-Dimensional $\mathcal{N}=(8,8) \mathrm{SYM}$}

Next, we introduce mass terms to the theory presented in the previous section with preserving two supercharges. The $Q_{ \pm}^{(0)}$-transformation (10)-(13) itself is deformed 
by a mass parameter $M$ as

$$
Q_{ \pm}=Q_{ \pm}^{(0)}+\Delta Q_{ \pm}
$$

with nontrivial transformation of $\Delta Q_{ \pm}$given by

$$
\begin{aligned}
& \Delta Q_{ \pm} \tilde{H}_{\mu}=\frac{M}{3} \psi_{ \pm \mu}, \quad \Delta Q_{ \pm} \tilde{h}_{i}=\frac{M}{3} \rho_{ \pm i}, \quad \Delta Q_{ \pm} H_{\mathrm{A}}=\frac{M}{3} \chi_{ \pm \mathrm{A}}, \\
& \Delta Q_{ \pm} \eta_{ \pm}=\frac{2 M}{3} \phi_{ \pm}, \quad \Delta Q_{\mp} \eta_{ \pm}= \pm \frac{M}{3} C .
\end{aligned}
$$

Then, the deformed supercharges $Q_{ \pm}$remain to be "nilpotent" up to gauge and $S U(2)_{R}$ transformations:

$$
\begin{aligned}
Q_{+}^{2} & \left.=\text { (infinitesimal gauge transformation by } \phi_{+}\right)+\frac{M}{3} J_{++}, \\
Q_{-}^{2} & =\left(\text { infinitesimal gauge transformation by }-\phi_{-}\right)-\frac{M}{3} J_{--}, \\
\left\{Q_{+}, Q_{-}\right\} & =\text {(infinitesimal gauge transformation by } C)-\frac{M}{3} J_{0} .
\end{aligned}
$$

Note that $\left(Q_{+}, Q_{-}\right)$is still a doublet under the $S U(2)_{R}$.

The mass deformed action we consider is

$$
\begin{aligned}
& S_{M}=\left(Q_{+} Q_{-}-\frac{M}{3}\right) \mathcal{F}_{M}, \quad \mathcal{F}_{M}=\mathcal{F}_{0}+\Delta F \\
& \Delta \mathcal{F}=\frac{1}{g_{2 d}^{2}} \int d^{2} x \operatorname{tr}\left[\sum_{\mathrm{A}=1}^{3} \frac{a_{\mathrm{A}}}{2} B_{\mathrm{A}}^{2}+\sum_{i=3}^{4} \frac{c_{i}}{2} X_{i}^{2}\right] .
\end{aligned}
$$

When coefficients $a_{\mathrm{A}}, c_{i}$ all fall into the interval $\left(-\frac{2 M}{3}, 0\right)$, scalars $X_{i}, B_{\mathrm{A}}$ have positive mass terms. For convenience, let us take

$$
a_{1}=a_{2}=a_{3}=-\frac{2 M}{9}, \quad c_{3}=c_{4}=-\frac{4 M}{9}
$$

in the followings. Because the similar relations to (17) hold for the deformed supercharges, $S_{M}$ is turned out to be invariant under the $Q_{ \pm}$transformations:

$$
Q_{ \pm} S_{M}=0
$$

The explicit form of the action is expressed as

$$
S_{M}=S_{0}+\Delta S,
$$

where

$$
\begin{aligned}
\Delta S= & \frac{1}{g_{2 d}^{2}} \int d^{2} x \operatorname{tr}\left[\frac{2 M^{2}}{81}\left(B_{A}^{2}+X_{i}^{2}\right)+\frac{M^{2}}{9}\left(\frac{C^{2}}{4}+\phi_{+} \phi_{-}\right)-\frac{M}{2} C\left[\phi_{+}, \phi_{-}\right]\right. \\
& +\frac{2 M}{3} \psi_{+\mu} \psi_{-\mu}+\frac{2 M}{9} \rho_{+i} \rho_{-i}+\frac{4 M}{9} \chi_{+A} \chi_{-A}-\frac{M}{6} \eta_{+} \eta_{-} \\
& \left.-\frac{4 i M}{9} B_{3}\left(F_{12}+i\left[X_{3}, X_{4}\right]\right)\right] .
\end{aligned}
$$


The first line and the third line in (27) give mass terms to scalars and to fermions, respectively. The second line represents the so called Myers term. ${ }^{29}$ Thanks to these terms, a fuzzy $S^{2}$ configuration satisfying

$$
\begin{aligned}
& {\left[\phi_{+}, \phi_{-}\right]=\frac{M}{3} C, \quad\left[C, \phi_{ \pm}\right]= \pm \frac{2 M}{3} \phi_{ \pm},} \\
& B_{\mathrm{A}}=X_{i}=0
\end{aligned}
$$

gives the minimum of the action $\left(S_{M}=0\right)$ preserving the $Q_{ \pm}$supersymmetries. Note that the contribution of the last line in (27) is not real, but pure imaginary. Also, we should recognize that the mass-deformed action preserves only two supersymmetries $\left(Q_{ \pm}\right)$but the other fourteen supersymmetries are broken by the deformation. So, the action may look somewhat similar to the action of PP wave matrix strings preserving eight supersymmetries, ${ }^{30}$ but it is different.

For various SYM models, this kind of mass deformation is discussed in Ref. 31.

\section{Lattice Formulation of Mass-Deformed Theory}

Now we present a lattice formulation for the mass-deformed theory in the previous section. On the two-dimensional square lattice $\mathbf{Z}^{2}$, gauge fields are promoted to $G=U(N)$ or $S U(N)$ group variables:

$$
A_{\mu}(x) \Rightarrow U_{\mu}(x)=e^{i a A_{\mu}(x)},
$$

where $U_{\mu}(x)$ is defined on the link $(x, x+\hat{\mu})$ which connects the lattice sites $x$ and $x+\hat{\mu}$. $\hat{\mu}$ is a unit vector along the $\mu$-th direction on the lattice. All the other fields are put on sites. Lattice fields are dimensionless and they are related to the continuum counterparts by

$$
\begin{aligned}
& (\text { scalars })^{\text {lat }}=a(\text { scalars })^{\text {cont }}, \quad(\text { fermions })^{\text {lat }}=a^{3 / 2}(\text { fermions })^{\text {cont }}, \\
& \left.(\text { auxiliary fields })^{\text {lat }}=a^{2} \text { (auxiliary fields }\right)^{\text {cont }}, \quad Q_{ \pm}^{\text {lat }}=a^{1 / 2} Q_{ \pm}^{\text {cont }} .
\end{aligned}
$$

Also, dimensionless coupling constants on the lattice are

$$
g_{0}=a g_{2 d}, \quad M_{0}=a M .
$$

$Q_{ \pm}$supersymmetries can be realized on the lattice as

$$
\begin{aligned}
Q_{ \pm} U_{\mu}(x)= & i \psi_{ \pm \mu}(x) U_{\mu}(x), \\
Q_{ \pm} \psi_{ \pm \mu}(x)= & i \psi_{ \pm \mu}(x) \psi_{ \pm \mu}(x) \pm i D_{\mu} \phi_{ \pm}(x), \\
Q_{\mp} \psi_{ \pm \mu}(x)= & \frac{i}{2}\left\{\psi_{+\mu}(x), \psi_{-\mu}(x)\right\}+\frac{i}{2} D_{\mu} C(x) \mp \tilde{H}_{\mu}(x), \\
Q_{ \pm} \tilde{H}_{\mu}(x)= & -\frac{1}{2}\left[\psi_{\mp \mu}(x), \phi_{ \pm}(x)+U_{\mu}(x) \phi_{ \pm}(x+\hat{\mu}) U_{\mu}(x)^{\dagger}\right] \\
& \pm \frac{1}{4}\left[\psi_{ \pm \mu}(x), C(x)+U_{\mu}(x) C(x+\hat{\mu}) U_{\mu}(x)^{\dagger}\right] \\
& \mp \frac{i}{2} D_{\mu} \eta_{ \pm}(x) \pm \frac{1}{4}\left[\psi_{ \pm \mu}(x) \psi_{ \pm \mu}(x), \psi_{\mp \mu}(x)\right] \\
& +\frac{i}{2}\left[\psi_{ \pm \mu}(x), \tilde{H}_{\mu}(x)\right]+\frac{M_{0}}{3} \psi_{ \pm \mu},
\end{aligned}
$$


where $D_{\mu} A(x) \equiv U_{\mu}(x) A(x+\hat{\mu}) U_{\mu}(x)^{-1}-A(x)$ are forward covariant differences for an adjoint field $A(x)$. The transformation rules for the other lattice fields are of the same form as in the continuum with the trivial replacement $M \rightarrow M_{0}$. Then, the "nilpotency" (22) is realized on the lattice.

In order to construct the corresponding lattice action, let us define a lattice counterpart of $\Phi_{\mathrm{A}}$ in $(9)$ as

$$
\begin{aligned}
& \Phi_{1}(x)=2\left(-D_{1} X_{3}(x)-D_{2} X_{4}(x)\right) \\
& \Phi_{2}(x)=2\left(-D_{1}^{*} X_{4}(x)+D_{2}^{*} X_{3}(x)\right) \\
& \Phi_{3}(x)=\frac{i\left(U_{12}(x)-U_{21}(x)\right)}{1-\epsilon^{-2}\left\|1-U_{12}(x)\right\|^{2}}+2 i\left[X_{3}(x), X_{4}(x)\right] .
\end{aligned}
$$

Here, $D_{\mu}^{*} A(x) \equiv A(x)-U_{\mu}(x-\hat{\mu})^{-1} A(x-\hat{\mu}) U_{\mu}(x-\hat{\mu})$ are backward covariant differences,

$$
U_{\mu \nu}(x)=U_{\mu}(x) U_{\nu}(x+\hat{\mu}) U_{\mu}(x+\hat{\nu})^{-1} U_{\nu}(x)^{-1}
$$

are plaquette variables, and we take $\|A\|=\sqrt{\operatorname{tr}\left(A A^{\dagger}\right)}$ as the norm of an arbitrary matrix $A . \epsilon$ is a positive number chosen as ${ }^{\mathrm{c}}$

$$
\begin{array}{cl}
0<\epsilon<2 & \text { for } G=U(N) \\
0<\epsilon<2 \sqrt{2} & \text { for } G=S U(N) \text { with } N=2,3,4 \\
0<\epsilon<2 \sqrt{N} \sin \frac{\pi}{N} & \text { for } G=S U(N) \text { with } N \geq 5 .
\end{array}
$$

The first term of the RHS of $\Phi_{3}(x)$ is a lattice counterpart of the field strength $F_{12}$. It is the same as the situation in the lattice formulation for two-dimensional $\mathcal{N}=(2,2) U(N)$ SYM $^{8}$ Then, $Q_{ \pm}$-invariant lattice action is given as

$$
S_{\text {lat }}=\left(Q_{+} Q_{-}-\frac{M_{0}}{3}\right) \mathcal{F}_{\text {lat }}
$$

with $\mathcal{F}_{\text {lat }}$ being the same form as $\mathcal{F}_{M}$ in (22) under the trivial replacement

$$
\frac{1}{g_{2 d}^{2}} \int d^{2} x \rightarrow \frac{1}{g_{0}^{2}} \sum_{x}, \quad M \rightarrow M_{0}
$$

when the admissibility condition $\left\|1-U_{12}(x)\right\|<\epsilon$ is satisfied for ${ }^{\forall} x$. Otherwise,

$$
S_{\text {lat }}=+\infty
$$

i.e. the Boltzmann weight $e^{-S_{\text {lat }}}$ vanishes.

c Argument deriving the result for $G=S U(N)$ in Ref. 8 has some error, although the final result is correct. For correct argument in the case $G=S U(N)$, see Ref. 32 . 


\subsection{Minimum of the lattice action}

Here, we will check that the lattice action has the minimum only at the pure gauge configuration $U_{12}(x)=\mathbb{1}_{N}$, which guarantees that the weak field expansion

$$
U_{\mu}(x)=1+i a A_{\mu}(x)+\frac{(i a)^{2}}{2 !} A_{\mu}(x)^{2}+\cdots
$$

is allowed in the continuum limit and that the lattice theory converges to the desired continuum theory at the classical level.

After integrating out the auxiliary fields, bosonic part of the action $S_{\text {lat }}$ takes the form

$$
\begin{aligned}
S_{\text {lat }}^{(B)}= & \frac{1}{g_{0}^{2}} \sum_{x} \operatorname{tr}\left[\frac{2 M_{0}^{2}}{81}\left(X_{i}(x)^{2}+B_{\mathrm{A}}(x)^{2}\right)\right. \\
& \left.-i \frac{4 M_{0}}{9} B_{3}(x)\left\{-\frac{1}{2} \frac{i\left(U_{12}(x)-U_{21}(x)\right)}{1-\frac{1}{\epsilon^{2}}\left\|1-U_{12}(x)\right\|^{2}}+i\left[X_{3}(x), X_{4}(x)\right]\right\}\right]+S_{\mathrm{PDT}}
\end{aligned}
$$

with $S_{\text {PDT }}$ representing positive semi-definite terms. We will treat the terms in the second line in (40), which is pure imaginary, as operators by employing the reweighting method. Then, for the remaining part of $S_{\text {lat }}^{(B)}$ we find that the mass terms in the first line fix the minimum at

$$
X_{i}(x)=B_{\mathrm{A}}(x)=0,
$$

which is independent of $S_{\mathrm{PDT}}$. At (41), $S_{\mathrm{PDT}}$ becomes

$$
\begin{aligned}
S_{\mathrm{PDT}}= & \frac{1}{g_{0}^{2}} \sum_{x} \operatorname{tr}\left[\sum_{\mu}\left(D_{\mu} X_{p}(x)\right)^{2}+\left(i\left[X_{p}(x), X_{q}(x)\right]+\frac{M_{0}}{3} \epsilon_{p q r} X_{r}(x)\right)^{2}\right] \\
& +\frac{1}{4 g_{0}^{2}} \sum_{x} \frac{\operatorname{tr}\left[-\left(U_{12}(x)-U_{21}(x)\right)^{2}\right]}{\left(1-\frac{1}{\epsilon^{2}}\left\|1-U_{12}(x)\right\|^{2}\right)^{2}}
\end{aligned}
$$

with $C=2 X_{8}, \phi_{ \pm}=X_{9} \pm i X_{10}$ and $p, q, r=8,9,10$. Notice that the last term representing the gauge kinetic term has the same form as in the case of two-dimensional $\mathcal{N}=(2,2) \mathrm{SYM}^{8}{ }^{8}$ where the admissibility condition (36) singles out the minimum $U_{12}(x)=\mathbb{1}_{N}$. In order to illustrate it, suppose we forget the admissibility condition $\left\|1-U_{12}(x)\right\|<\epsilon$ for a moment in the case $G=U(N)$. The action has the minimum at configurations satisfying

$$
U_{12}(x)^{2}+U_{21}(x)^{2}=2
$$

namely

$$
U_{12}(x)=\left(\begin{array}{lll} 
\pm 1 & & \\
& \ddots & \\
& & \pm 1
\end{array}\right)
$$

up to gauge transformations with all the combinations of \pm 1 in the diagonal entries allowed. Then, we should take into account fluctuations around each configuration 
of (44) although only the single configuration $U_{12}(x)=\mathbb{1}_{N}$ allows the weak field expansion (39) leading to the target continuum theory. However, by imposing the admissibility condition $\left\|1-U_{12}(x)\right\|<\epsilon$, all the configurations of (44) are excluded except $U_{12}(x)=\mathbb{1}_{N}$. In this way, we can successfully single out the configuration $U_{12}(x)=\mathbb{1}_{N}$. Notice that the standard Wilson's lattice gauge action has the minimum at

$$
U_{12}(x)+U_{21}(x)=2
$$

leading to $U_{12}(x)=\mathbb{1}_{N}$. "The square" of $U_{12}(x)$ or $U_{21}(x)$ in (43) is a crucial difference from the Wilson case (45).

Hence, we obtain the single minimum $U_{12}(x)=\mathbb{1}_{N}$ for the action $S_{\text {lat }}$. The mass deformation preserving $Q_{ \pm}$is important to stabilize flat directions of scalars as well as to remove degeneracy of gauge fields.

\subsection{Absence of doubler modes}

In order to check that no doubler appears in the lattice action $S_{\text {lat }}$, let us set $U_{\mu}(x)=\mathbb{1}_{N}$ and pick up quadratic kinetic terms.

Then, we have the kinetic terms for scalars

$$
\begin{aligned}
S_{\text {lat }}^{(2, B)}= & \frac{1}{g_{0}^{2}} \sum_{x} \operatorname{tr}\left[\frac{2 M_{0}^{2}}{81}\left(\sum_{i} X_{i}(x)^{2}+\sum_{\mathrm{A}} B_{\mathrm{A}}(x)^{2}\right)\right. \\
& +\sum_{\mu}\left\{\Delta_{\mu} \phi(x) \Delta_{\mu} \bar{\phi}(x)+\frac{1}{4}\left(\Delta_{\mu} C(x)\right)^{2}\right\} \\
& +\frac{M_{0}^{2}}{9}\left(\frac{1}{4} C(x)^{2}+\phi(x) \bar{\phi}(x)\right) \\
& +\left(\Delta_{1}^{*} B_{1}(x)-\Delta_{2} B_{2}(x)\right)^{2}+\left(\Delta_{2}^{*} B_{1}(x)+\Delta_{1} B_{2}(x)\right)^{2} \\
& +\left(\Delta_{1} X_{3}(x)+\Delta_{2} X_{4}(x)\right)^{2}+\left(-\Delta_{1}^{*} X_{4}(x)+\Delta_{2}^{*} X_{3}(x)\right)^{2} \\
& \left.+\sum_{\mu}\left(\Delta_{\mu}^{*} B_{3}(x)\right)^{2}\right]
\end{aligned}
$$

where $\Delta_{\mu}\left(\Delta_{\mu}^{*}\right)$ are forward (backward) difference operators:

$$
\Delta_{\mu} f(x)=f(x+\hat{\mu})-f(x), \quad \Delta_{\mu}^{*} f(x)=f(x)-f(x-\hat{\mu}) .
$$

Using the relation

$$
\sum_{x} f(x) \Delta_{\mu} g(x)=-\sum_{x}\left(\Delta_{\mu}^{*} f(x)\right) g(x)
$$


we find that (46) takes the form of the standard kinetic terms for scalars:

$$
\begin{aligned}
S_{\mathrm{lat}}^{(2, B)}= & \frac{1}{g_{0}^{2}} \sum_{x} \operatorname{tr}\left[\sum_{\mu} \Delta_{\mu} \phi(x) \Delta_{\mu} \bar{\phi}(x)+\frac{M_{0}^{2}}{9} \phi(x) \bar{\phi}(x)\right. \\
& +\frac{1}{4}\left\{\sum_{\mu}\left(\Delta_{\mu} C(x)\right)^{2}+\frac{M_{0}^{2}}{9} C(x)^{2}\right\} \\
& +\sum_{\mathrm{A}}\left\{\sum_{\mu}\left(\Delta_{\mu} B_{\mathrm{A}}(x)\right)^{2}+\frac{2 M_{0}^{2}}{81} B_{\mathrm{A}}(x)^{2}\right\} \\
& \left.+\sum_{i}\left\{\sum_{\mu}\left(\Delta_{\mu} X_{i}(x)\right)^{2}+\frac{2 M_{0}^{2}}{81} X_{i}(x)^{2}\right\}\right],
\end{aligned}
$$

indicating that no doubler appears in the bosonic sector.

For fermions, the kinetic terms can be expressed in the form:

$$
\begin{aligned}
S_{\text {lat }}^{(2, F)}= & \frac{1}{g_{0}^{2}} \sum_{x} \operatorname{tr}\left[\sum_{\mu} \Psi^{(0)}(x)^{T} G_{\mu} \frac{1}{2}\left(\Delta_{\mu}+\Delta_{\mu}^{*}\right) \Psi^{(0)}(x)\right. \\
& +\sum_{\mu} \Psi^{(0)}(x)^{T} P_{\mu} \frac{1}{2}\left(\Delta_{\mu}-\Delta_{\mu}^{*}\right) \Psi^{(0)}(x) \\
& \left.+\Psi^{(0)}(x)^{T} \mathcal{M} \Psi^{(0)}(x)\right]
\end{aligned}
$$

where $\Psi^{(0)}$ is given in Eq. (A.3), $G_{\mu}$ are $16 \times 16$ imaginary symmetric (antihermitian) matrices and $P_{\mu}$ are $16 \times 16$ imaginary anti-symmetric (hermitian) matrices, satisfying

$$
\left\{G_{\mu}, G_{\nu}\right\}=-2 \delta_{\mu \nu} \mathbb{1}_{16}, \quad\left\{P_{\mu}, P_{\nu}\right\}=2 \delta_{\mu \nu} \mathbb{1}_{16}, \quad\left\{G_{\mu}, P_{\nu}\right\}=0 .
$$

The mass matrix is

$$
\mathcal{M}=\frac{M_{0}}{9}\left(\begin{array}{c}
m_{d} \\
-m_{d}
\end{array}\right), \quad m_{d}=\operatorname{diag}(1,1,3,3,2,2,2,-3) .
$$

When we move to the momentum space via

$$
\Psi^{(0)}(x)=\int_{-\pi / a}^{\pi / a} \frac{d^{2} p}{(2 \pi)^{2}} \tilde{\Psi}^{(0)}(p) e^{i a p \cdot x},
$$

the kernel

$$
\mathcal{D}_{F}=\sum_{\mu}\left[G_{\mu} \frac{1}{2}\left(\Delta_{\mu}+\Delta_{\mu}^{*}\right)+P_{\mu} \frac{1}{2}\left(\Delta_{\mu}-\Delta_{\mu}^{*}\right)\right]
$$

is expressed as

$$
\tilde{\mathcal{D}}_{F}(p)=\sum_{\mu=1}^{2}\left[i G_{\mu} \sin \left(a p_{\mu}\right)-2 P_{\mu} \sin ^{2}\left(\frac{a p_{\mu}}{2}\right)\right] .
$$


Using (51), we can easily see

$$
\tilde{\mathcal{D}}_{F}(p)^{2}=\sum_{\mu=1}^{2} 4 \sin ^{2}\left(\frac{a p_{\mu}}{2}\right) .
$$

Since $\tilde{\mathcal{D}}_{F}(p)$ is hermitian, (56) shows that only the origin $\left(p_{1}, p_{2}\right)=(0,0)$ gives the zero of $\tilde{\mathcal{D}}_{F}(p)$. It proves that there appears no doubler in the fermionic sector.

\subsection{No need of fine-tuning}

We give a perturbative argument to show that the lattice action converges to the desired continuum theory in the quantum mechanical sense without any fine-tuning.

After integrating out the auxiliary fields in the theory near the continuum limit, let us consider local operators of the type:

$$
\mathcal{O}_{p}(x)=M^{m} \varphi(x)^{\alpha} \partial^{\beta} \psi(x)^{2 \gamma},
$$

where $m, \alpha, \beta, \gamma=0,1,2, \ldots$, and $\partial$ means derivative operators. $\varphi(x)$ and $\psi(x)$ represent a general bosonic field and a general fermionic field, respectively. The mass dimension of $\mathcal{O}_{p}(x)$ is $p \equiv m+\alpha+\beta+3 \gamma$. Dimensional analysis tells that radiative corrections from UV region of loop momenta to $\mathcal{O}_{p}(x)$ has the form

$$
\left(\frac{1}{g_{2 d}^{2}} c_{0} a^{p-4}+c_{1} a^{p-2}+g_{2 d}^{2} c_{2} a^{p}+\cdots\right) \int d^{2} x \mathcal{O}_{p}(x)
$$

up to possible powers of $\ln (a M) . c_{0}, c_{1}, c_{2}$ are dimensionless numerical constants. The first, second and third terms in the parenthesis are contributions from tree, 1-loop and 2-loop effects, respectively. The "..." is a contribution from higher loops, which is irrelevant for the analysis here. Since relevant or marginal operators generated by loop effects possibly appear from nonpositive powers of $a$ in the second and third terms, we should see operators with $p=0,1,2$. They are $\varphi, M \varphi$ and $\varphi^{2}$. (Note that $\mathbb{1}, M, M^{2}$ and $\partial \varphi$ are not dynamical.) For $G=U(N)$ case, candidates for $\varphi$ are linear combinations of $\operatorname{tr} X_{i}$ and $\operatorname{tr} B_{\mathrm{A}}$ from gauge and $S U(2)_{R}$ symmetries. But, all of them are not invariant under $Q_{ \pm}$supersymmetries, and thus are forbidden to appear. Similarly we can show that $M \varphi$ and $\varphi^{2}$ are not allowed to be generated. For $G=S U(N)$, we may consider $\operatorname{tr} \varphi^{2}$ alone, whose candidates are not generated due to the symmetries.

Hence, we can conclude that any relevant or marginal operators except nondynamical constant operators are not radiatively generated. It is shown that no fine-tuning is required in taking the continuum limit.

\section{Matrix String Theory}

The mass-deformed $\mathcal{N}=(8,8)$ SYM with $G=U(N)$ in two dimensions can be obtained from the corresponding lattice theory around the trivial minimum $C=$ $\phi_{ \pm}=0$ as seen in the previous section. Since $M$ is a soft mass breaking sixteen 
supersymmetries to $Q_{ \pm}$, the undeformed theory with $G=U(N)$, which is nothing but IIA matrix string theory, ${ }^{3}$ can be defined by turning off $M$ after the continuum limit.

\section{Four-Dimensional $\mathcal{N}=4 \mathrm{SYM}$}

In this section, we discuss a scenario to obtain four-dimensional $\mathcal{N}=4 \mathrm{SYM}$ from the lattice formulation given in section 4 with $G=U(N)$.

Let us consider the lattice theory expanded around the minimum of $k$-coincident fuzzy $S^{2}$ :

$$
C=\frac{2 M_{0}}{3} L_{3}, \quad \phi_{ \pm}=\frac{M_{0}}{3}\left(L_{1} \pm i L_{2}\right)
$$

with

$$
L_{a}=L_{a}^{(n)} \otimes \mathbb{1}_{k} \quad(a=1,2,3) \quad \text { and } \quad N=n k .
$$

$L_{a}^{(n)}$ are $S U(2)$-generators of an $n(=2 j+1)$-dimensional irreducible representation of $\operatorname{spin} j$.

First, we take the continuum limit of the two-dimensional lattice theory with $G=U(N)$. Then, we obtain four-dimensional $\mathcal{N}=4 U(k)$ SYM on $\mathbf{R}^{2} \times\left(\right.$ Fuzzy $\left.S^{2}\right)$ with sixteen supercharges broken to $Q_{ \pm}$by $M{ }^{\mathrm{d}}$ The fuzzy $S^{2}$ has the radius $R=\frac{3}{M}$, and its noncommutativity (fuzziness) is characterized by the parameter $\Theta=\frac{18}{M^{2} n}$. UV cutoff in the $S^{2}$ directions is $\Lambda=\frac{M}{3} \cdot 2 j$. These properties of the fuzzy $S^{2}$ are seen by doing a similar calculation as presented in Refs. 22, 24, 33. In particular, momentum modes of a field, say $B_{\mathrm{A}}$, on two dimensions are expanded further by fuzzy spherical harmonics:

$$
\tilde{B}_{\mathrm{A}}(q)=\sum_{J=0}^{2 j} \sum_{m=-J}^{J} \hat{Y}_{J m}^{(j j)} \otimes b_{\mathrm{A}, J m}
$$

corresponding to the expression (60). The fuzzy spherical harmonic $\hat{Y}_{J m}^{(j j)}$ is an $n \times n$ matrix whose elements are given by Clebsch-Gordon coefficients as ${ }^{24}$

$$
\hat{Y}_{J m}^{(j j)}=\sqrt{n} \sum_{r, r^{\prime}=-j}^{j}(-1)^{-j+r^{\prime}} C_{j r j-r^{\prime}}^{J m}|j r\rangle\left\langle j r^{\prime}\right|
$$

with an orthonormal basis $|j r\rangle$ representing $L_{a}^{(n)}$ in the standard way:

$$
\begin{aligned}
\left(L_{1}^{(n)} \pm i L_{2}^{(n)}\right)|j r\rangle & =\sqrt{(j \mp r)(j \pm r+1)}|j r \pm 1\rangle, \\
L_{3}^{(n)}|j r\rangle & =r|j r\rangle,
\end{aligned}
$$

d Due to the infinite volume of $\mathbf{R}^{2}$, tunnelling among discrete minima of various fuzzy sphere solutions is suppressed to stabilize each fuzzy sphere background. 
and the modes $b_{\mathrm{A}, J} m$ are $k \times k$ matrices. It is seen that the fuzzy spherical harmonics are eigen-modes of the Laplacian on the fuzzy $S^{2}$ :

$$
\sum_{a=1}^{3}\left(\frac{M}{3}\right)^{2}\left[L_{a}^{(n)},\left[L_{a}^{(n)}, \hat{Y}_{J m}^{(j j)}\right]\right]=\left(\frac{M}{3}\right)^{2} J(J+1) \hat{Y}_{J m}^{(j j)},
$$

giving the rotational energy with the angular momentum $J$ on the sphere of the radius $R=\frac{3}{M}$. The UV cutoff $\Lambda=\frac{M}{3} \cdot 2 j$ can be read off from the upper limit of the sum of $J$ in the expansion (61). The fuzzy $S^{2}$ is a two-dimensional noncommutative (NC) space, which is analogous to the phase space of some one-dimensional quantum system, and the noncommutativity $\Theta$ to the Planck constant $\hbar$. The quantum phase space is divided into small cells of the size $2 \pi \hbar$, whose number is equal to the dimension of the Hilbert space. Correspondingly, the area of the $S^{2}$ is divided into $n$ cells of the size $2 \pi \Theta$ :

$$
4 \pi R^{2}=n \cdot 2 \pi \Theta
$$

leading to the value $\Theta=\frac{18}{M^{2} n}$.

Notice, differently from the two-dimensional case, it is not clear whether the supersymmetry breaking by $M$ is soft, because $M$ appears not only in mass terms in the action but also in the geometry of the fuzzy $S^{2}$. Let us proceed assuming that the breaking is soft. ${ }^{\mathrm{e}}$ We will give some argument later for the validity of the assumption.

Next, we take successive limits by following the two steps:

- Step 1: Take large $n$ limit with $\Theta$ and $k$ fixed. Namely, $M \propto n^{-1 / 2} \rightarrow 0$ and $\Lambda \propto n^{1 / 2} \rightarrow \infty$.

- Step 2: Send $\Theta$ to zero.

\subsection{Step 1}

At the step 1, the fuzzy $S^{2}$ is decompactified to the NC Moyal plane $\mathbf{R}_{\Theta}^{2}$. From the assumption, the theory becomes $\mathcal{N}=4 U(k)$ SYM on $\mathbf{R}^{2} \times \mathbf{R}_{\Theta}^{2}$ with sixteen supersymmetries restored. The gauge coupling constant of the four-dimensional theory is given in the form

$$
g_{4 d}^{2}=2 \pi \Theta g_{2 d}^{2}
$$

In the limit, the expansion (61) by the fuzzy spherical harmonics can be essentially transcribed to the one by plane waves on $\mathbf{R}_{\Theta}^{2}$ :

$$
\tilde{B}_{\mathrm{A}}(q)=\int \frac{d^{2} \tilde{q}}{(2 \pi)^{2}} e^{i \tilde{q} \cdot \hat{x}} \otimes \tilde{b}_{\mathrm{A}}(\mathbf{q})
$$

e The assumption is plausible from the viewpoint of the mapping rule between matrix model and Yang-Mills theory on noncommutative space. ${ }^{34}$ 
where $q$ and $\tilde{q}$ are two-momenta on $\mathbf{R}^{2}$ and $\mathbf{R}_{\Theta}^{2}$ respectively, the position operator $\hat{x}=\left(\hat{x}_{1}, \hat{x}_{2}\right)$ on $\mathbf{R}_{\Theta}^{2}$ satisfies $\left[\hat{x}_{1}, \hat{x}_{2}\right]=i \Theta$, and $\mathbf{q} \equiv(q, \tilde{q})$ represents a fourmomentum. The modes $\tilde{b}_{\mathrm{A}}(\mathbf{q})$ in the four-dimensional space are $k \times k$ matrices. It is easy to calculate the inner product between plane waves on $\mathbf{R}_{\Theta}^{2}$ :

$$
\operatorname{Tr}\left(e^{i \tilde{p} \cdot \hat{x}} e^{i \tilde{q} \cdot \hat{x}}\right)=\frac{2 \pi}{\Theta} \delta^{2}(\tilde{p}+\tilde{q}),
$$

which leads to the $\Theta$-dependence of the relation (66).

Let us discuss radiative corrections in four-dimensional SYM on $\mathbf{R}^{2} \times\left(\right.$ Fuzzy $\left.S^{2}\right)$. We give an argument below that there is no radiative correction which prevents from the full sixteen supersymmetries being restored after the step 1.

In quantum field theory defined on NC space with a constant noncommutativity, there are two kinds of Feynman diagrams. One is planar diagrams. They have no NC phase factors depending on loop momenta, and their behavior is the same as that in the corresponding theory on the ordinary space. ${ }^{35}$ The other is nonplanar diagrams. They have NC phase factors, which improve the UV behavior of the diagrams. But, when some of the NC phases vanish in the infra-red (IR) region of external or loop momenta, singularities may arise, whose origin is the UV singularities in the corresponding theory on the ordinary space (UV/IR mixing). ${ }^{36}$ Therefore, we can say that UV behavior of planar and nonplanar diagrams in the theory on NC space is not worse than that in the corresponding theory on the ordinary space. Let us consider the superficial degree of UV divergences of Feynman diagrams in ordinary four-dimensional gauge theory:

$$
D=4-E_{B}-\frac{3}{2} E_{F}
$$

where $E_{B}\left(E_{F}\right)$ is the number of the external lines of bosons (fermions). In our case, the divergence of $D=3$, that is from $E_{B}=1$, is absent since the operator $\varphi$ is forbidden by $Q_{ \pm}$supersymmetries as in the two-dimensional case. Thus, the possible most severe divergences are of the degree $D=2$. The leading $\Lambda^{2}$ terms are expected to cancel each other by sixteen supersymmetries under the assumption that $M$ is soft. For radiative corrections to gauge invariant observables, divergences possibly originate from the mass deformation, whose behavior is expected $\operatorname{as}^{\mathrm{f}}$

$$
M^{p}\left(\ln \frac{\Lambda}{M}\right)^{q}=\mathcal{O}\left(M^{p}(\ln n)^{q}\right) \quad(p=1,2, q=1,2, \ldots) .
$$

However, such terms disappear in the limit of the step 1.

Hence, there appears no radiative correction preventing restoration of the full supersymmetries after the step 1 , leading to $\mathcal{N}=4 U(k)$ SYM on $\mathbf{R}^{2} \times \mathbf{R}_{\Theta}^{2}$ with sixteen supercharges.

$\mathrm{f}$ We should note that the behavior (70) is not valid for gauge-dependent divergences which can be absorbed into wave function renormalization. (70) is derived based on UV finiteness of the undeformed four-dimensional $\mathcal{N}=4 \mathrm{SYM},{ }^{37,}, 38$ where the finiteness holds except such divergences. 


\subsection{Step 2}

In four-dimensional $\mathcal{N}=4 \mathrm{SYM}$ on $\mathrm{NC}$ space, the commutative limit $(\Theta \rightarrow 0)$ is believed to be smooth, ${ }^{39,40}$ that is, desired $\mathcal{N}=4 U(k)$ SYM on usual flat $\mathbf{R}^{4}$ should be obtained with no fine tuning after the step 2 .

\subsection{Check of the scenario}

As a check of the scenario presented in the above, we carry out one-loop computation of radiative corrections from the both of planar and nonplanar diagrams to scalar kinetic terms of $X_{i}(i=3,4)$ and $B_{\mathrm{A}}(\mathrm{A}=1,2)$ in $\mathcal{N}=4 U(k) \mathrm{SYM}$ on $\mathbf{R}^{2} \times$ (Fuzzy $S^{2}$ ), and take the limits of Steps 1 and 2 .

The final expression turns out to be consistent to the standard one-loop result of $\mathcal{N}=4 \mathrm{SYM}$ on $\mathbf{R}^{4}$. In particular, the overall $U(1)$ part receives no radiative correction and becomes free. The $S U(k)$ part of the kinetic terms is

$$
\begin{aligned}
& \frac{1}{g_{4 d}^{2}} \int \frac{d^{4} \mathbf{q}}{(2 \pi)^{4}} \mathbf{q}^{2}\left\{\sum_{\mathrm{A}=1,2} \operatorname{tr}_{k}\left[\tilde{b}_{\mathrm{A}}^{(R)}(-\mathbf{q}) \tilde{b}_{\mathrm{A}}^{(R)}(\mathbf{q})\right]+\sum_{i=3,4} \operatorname{tr}_{k}\left[\tilde{x}_{i}^{(R)}(-\mathbf{q}) \tilde{x}_{i}^{(R)}(\mathbf{q})\right]\right\} \\
& \quad \times\left\{1+\frac{g_{4 d}^{2} k}{4 \pi^{2}}\left(-\frac{1}{2} \ln \frac{\mathbf{q}^{2}}{\mu_{R}^{2}}+1\right)+\mathcal{O}\left(g_{4 d}^{4}\right)\right\}
\end{aligned}
$$

after the limit of the step 1. $\tilde{b}_{\mathrm{A}}^{(R)}(\mathbf{q}), \tilde{x}_{i}^{(R)}(\mathbf{q})$ are renormalized momentum modes which are related to the modes $\tilde{b}_{\mathrm{A}}(\mathbf{q}), \tilde{x}_{i}(\mathbf{q})$ of the bare fields $B_{\mathrm{A}}, X_{i}$ as

$$
\tilde{b}_{\mathrm{A}}^{(R)}(\mathbf{q})=\left(1+\frac{g_{4 d}^{2} k}{4 \pi^{2}} \ln \frac{\Lambda}{\mu_{R}}\right)^{1 / 2} \tilde{b}_{\mathrm{A}}(\mathbf{q}), \quad \tilde{x}_{i}^{(R)}(\mathbf{q})=\left(1+\frac{g_{4 d}^{2} k}{4 \pi^{2}} \ln \frac{\Lambda}{\mu_{R}}\right)^{1 / 2} \tilde{x}_{i}(\mathbf{q}),
$$

where $\mu_{R}$ is the renormalization point. ${ }^{\mathrm{g}}$ The logarithmic nonlocal term in (71) has a definite physical meaning contributing to anomalous scaling dimension of the operator. Since the result does not depend on $\Theta$, the limit of the step 2 is trivial.

Note that singular behavior due to the UV/IR mixing does not survive in the limits, and that four-dimensional rotational symmetry is restored in (71), which can be regarded as an evidence of the restoration of the full sixteen supersymmetries and of the softness of $M$.

\section{Summary and Discussion}

Firstly, we gave a brief review of recent development of lattice formulations for supersymmetric gauge theories, where "nilpotent" supercharges not generating translations play a key role.

g Although four-dimensional $\mathcal{N}=4 \mathrm{SYM}$ is said to be UV finite, divergence of the wave function renormalization can appear as a gauge artifact. ${ }^{37}$ In fact, a modified light-cone gauge fixing in Refs. 37 leads to no UV divergence even in the part concerning the wave function renormalization, differently from the 1-loop computation in Feynman gauge fixing. ${ }^{38}$ The point is that even if UV divergences appear in radiative corrections, all of them can be absorbed by rescaling the fields. ${ }^{38}$ We adopted a Feynman-like gauge fixing in the calculation. 
Secondly, we presented a lattice formulation for mass-deformed $\mathcal{N}=(8,8) \mathrm{SYM}$ in two dimensions with the gauge groups $G=U(N), S U(N)$, which preserves two supercharges. Owing to the mass-deformation, flat directions of scalars are stabilized. It is advantagous in performing actual Monte Carlo simulation. Also, since the mass is soft, ordinary two-dimensional $\mathcal{N}=(8,8) \mathrm{SYM}$ is obtained by turning off the mass after the continuum limit. It is applicable to nonperturbative analysis of IIA matrix string theory. ${ }^{3}$

Thirdly, we discussed a scenario to obtain four-dimensional $\mathcal{N}=4 U(k) \mathrm{SYM}$ from the two-dimensional theory with $G=U(N)$ around a $k$-coincident fuzzy $S^{2}$ background. As a check of the scenario, we carried out explicit calculation of oneloop radiative corrections to the kinetic terms of $B_{\mathrm{A}}(\mathrm{A}=1,2)$ and $X_{i}(i=3,4)$ in the theory on $\mathbf{R}^{2} \times\left(\right.$ Fuzzy $\left.S^{2}\right)$. So far, the result suggests that the scenario works well. Although it requires more computation to establish the scenario, if it is correct, four-dimensional $\mathcal{N}=4 \mathrm{SYM}$ with gauge group $U(k)$ of finite rank can be defined nonperturbatively. It will give an intriguing tool to check AdS/CFT correspondence $^{4}$ in a regime where string loop effects cannot be neglected.

Fourthly, as discussed in Ref. 41, it is possible to deform two-dimensional $\mathcal{N}=$ $(4,4)$ SYM with $G=U(N), S U(N)$ by introducing a mass. Although it preserves all of the eight supercharges, not all flat directions of scalars are stabilized. Further deformation by another mass can be done in a manner to keep two supercharges, in order to lift up all the flat directions. We can put the theory deformed by two mass parameters on the lattice preserving the two supercharges. Four-dimensional theory constructed around a fuzzy $S^{2}$ background in the theory with $G=U(N)$ becomes $\mathcal{N}=2 U(k)$ SYM on $\mathbf{R}^{2} \times$ NC $\mathbf{R}^{2}$. Although the limit turning off $\Theta$ in this case is not expected to be smooth, it would be useful to investigate nonperturbative aspects of noncommutative $\mathcal{N}=2 \mathrm{SYM}$ in four dimensions.

Finally, it is also interesting to construct a similar lattice formulation for SQCD coupled with matter fields and for $\mathcal{N}=1^{*}, 2^{*}$ theories in four dimensions. In particular, cases leading to four-dimensional superconformal theories would be intriguing, where turning off the noncommutativity is expected to be smooth.

\section{Acknowledgments}

The author thanks Masanori Hanada, So Matsuura and Hiroshi Suzuki for the collaboration which is main ingredients for the presentation, and Chuan-Tsung Chan, Yasuaki Hikida, Satoshi Iso, Issaku Kanamori, Shoichi Kawamoto, Yoichi Kazama, Tsunehide Kuroki, Jun Nishimura, Kazumi Okuyama and Dan Tomino for useful and enjoyable discussions. Also, he thanks the organizers and all the participants of the conference for generating stimulating atmosphere.

\section{Appendix A. Notations}

We give the relation between field variables in (5) and (6), and the explicit form of the gamma matrices we used in (4). 
For scalars,

$$
B_{1}=-X_{5}, \quad B_{2}=X_{6}, \quad B_{3}=X_{7}, \quad C=2 X_{8}, \quad \phi_{ \pm}=X_{9} \pm i X_{10} .
$$

For fermionic variables,

$$
\begin{aligned}
& \Psi= U_{16} \Psi^{(0)}, \\
&\left(\Psi^{(0)}\right)^{T} \equiv\left(\rho_{+3}, \rho_{+4}, \psi_{+2}, \psi_{+1},-\chi_{+1}, \chi_{+2}, \chi_{+3}, \frac{1}{2} \eta_{+},\right. \\
&\left.\quad \rho_{-3}, \rho_{-4}, \psi_{-2}, \psi_{-1},-\chi_{-1}, \chi_{-2}, \chi_{-3}, \frac{1}{2} \eta_{-}\right),
\end{aligned}
$$

where $U_{16}$ is a $16 \times 16$ unitary matrix of the form

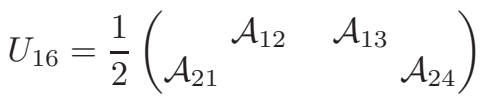

with

$$
\begin{aligned}
\mathcal{A}_{12} \equiv\left(\begin{array}{cccc}
0 & 0 & -i & 1 \\
i & 1 & 0 & 0 \\
0 & 0 & -1 & -i \\
1 & -i & 0 & 0 \\
i & -1 & 0 & 0 \\
0 & 0 & i & 1 \\
1 & i & 0 & 0 \\
0 & 0 & 1 & -i
\end{array}\right), \quad \mathcal{A}_{13} \equiv\left(\begin{array}{ccccc}
0 & 0 & -1 & i \\
-1 & i & 0 & 0 \\
0 & 0 & -i & -1 \\
-i & -1 & 0 & 0 \\
-1 & -i & 0 & 0 \\
0 & 0 & 1 & i \\
-i & 1 & 0 & 0 \\
0 & 0 & i & -1
\end{array}\right), \\
\mathcal{A}_{21} \equiv\left(\begin{array}{cccc}
0 & 0 & -1 & i \\
i & 1 & 0 & 0 \\
0 & 0 & -1 & i \\
-1 & i & 0 & 0 \\
i & -1 & 0 & 0 \\
0 & 0 & -i & 1 \\
-1 & -i & 0 & 0 \\
0 & 0 & 1 & i
\end{array}\right), \quad \mathcal{A}_{24} \equiv\left(\begin{array}{cccc}
1 & -i & 0 & 0 \\
0 & 0 & i & 1 \\
-i & -1 & 0 & 0 \\
1 & i & 0 & 0 \\
0 & 0 & 1 & i \\
-i & 1 & 0 & 0 \\
0 & 0 & -i & 1
\end{array}\right) .
\end{aligned}
$$

Also, the explicit form of the gamma matrices we used is

$$
\begin{aligned}
\gamma_{2} & =-i \mathbb{1}_{2} \otimes \mathbb{1}_{2} \otimes \mathbb{1}_{2} \otimes \sigma_{3}, \\
\gamma_{3} & =-i \mathbb{1}_{2} \otimes \mathbb{1}_{2} \otimes \mathbb{1}_{2} \otimes \sigma_{1}, \\
\gamma_{4} & =+i \mathbb{1}_{2} \otimes \mathbb{1}_{2} \otimes \sigma_{2} \otimes \sigma_{2}, \\
\gamma_{5} & =-i \sigma_{2} \otimes \sigma_{3} \otimes \sigma_{3} \otimes \sigma_{2}, \\
\gamma_{6} & =+i \sigma_{2} \otimes \mathbb{1}_{2} \otimes \sigma_{1} \otimes \sigma_{2}, \\
\gamma_{7} & =-i \sigma_{2} \otimes \sigma_{1} \otimes \sigma_{3} \otimes \sigma_{2}, \\
\gamma_{8} & =-i \sigma_{1} \otimes \sigma_{2} \otimes \sigma_{1} \otimes \sigma_{2}, \\
\gamma_{9} & =+i \mathbb{1}_{2} \otimes \sigma_{2} \otimes \sigma_{3} \otimes \sigma_{2}, \\
\gamma_{10} & =+i \sigma_{3} \otimes \sigma_{2} \otimes \sigma_{1} \otimes \sigma_{2}
\end{aligned}
$$

with $\sigma_{a}(a=1,2,3)$ being the Pauli matrices. 


\section{References}

1. T. Banks, W. Fischler, S. H. Shenker and L. Susskind, Phys. Rev. D 55, 5112 (1997), arXiv:hep-th/9610043.

2. N. Ishibashi, H. Kawai, Y. Kitazawa and A. Tsuchiya, Nucl. Phys. B 498, 467 (1997), arXiv:hep-th/9612115.

3. L. Motl, arXiv:hep-th/9701025; R. Dijkgraaf, E. P. Verlinde and H. L. Verlinde, Nucl. Phys. B 500, 43 (1997), arXiv:hep-th/9703030.

4. J. M. Maldacena, Adv. Theor. Math. Phys. 2, 231 (1998), arXiv:hep-th/9711200; N. Itzhaki, J. M. Maldacena, J. Sonnenschein and S. Yankielowicz, Phys. Rev. D 58, 046004 (1998), arXiv:hep-th/9802042.

5. S. Catterall, D. B. Kaplan and M. Ünsal, Phys. Rept. 484, 71 (2009), arXiv:0903.4881 [hep-lat].

6. A. Joseph, Int. J. Mod. Phys. A 26, 5057 (2011), arXiv:1110.5983 [hep-lat].

7. A. G. Cohen, D. B. Kaplan, E. Katz and M. Ünsal, J. High Energy Phys. 0308, 024 (2003), arXiv:hep-lat/0302017.

8. F. Sugino, J. High Energy Phys. 0403, 067 (2004), arXiv:hep-lat/0401017.

9. F. Sugino, Phys. Lett. B 635, 218 (2006), arXiv:hep-lat/0601024.

10. A. G. Cohen, D. B. Kaplan, E. Katz and M. Ünsal, J. High Energy Phys. 0312, 031 (2003), arXiv:hep-lat/0307012.

11. D. B. Kaplan and M. Ünsal, J. High Energy Phys. 0509, 042 (2005), arXiv:heplat/0503039.

12. M. G. Endres and D. B. Kaplan, J. High Energy Phys. 0610, 076 (2006), arXiv:heplat/0604012.

13. S. Matsuura, J. High Energy Phys. 0807, 127 (2008), arXiv:0805.4491 [hep-th].

14. F. Sugino, Nucl. Phys. B 808, 292 (2009), arXiv:0807.2683 [hep-lat].

15. Y. Kikukawa and F. Sugino, Nucl. Phys. B 819, 76 (2009), arXiv:0811.0916 [hep-lat].

16. D. Kadoh, F. Sugino and H. Suzuki, Nucl. Phys. B 820, 99 (2009), arXiv:0903.5398 [hep-lat].

17. I. Kanamori and H. Suzuki, Nucl. Phys. B 811, 420 (2009), arXiv:0809.2856 [hep-lat].

18. M. Hanada and I. Kanamori, Phys. Rev. D 80, 065014 (2009), arXiv:0907.4966 [heplat].

19. M. Hanada and I. Kanamori, J. High Energy Phys. 1101, 058 (2011), arXiv:1010.2948 [hep-lat].

20. M. Hanada, S. Matsuura and F. Sugino, Prog. Theor. Phys. 126, 597 (2011), arXiv:1004.5513 [hep-lat].

21. M. Hanada, S. Matsuura, F. Sugino and H. Suzuki, work in progress.

22. J. M. Maldacena, M. M. Sheikh-Jabbari and M. Van Raamsdonk, J. High Energy Phys. 0301, 038 (2003), arXiv:hep-th/0211139.

23. D. E. Berenstein, J. M. Maldacena and H. S. Nastase, J. High Energy Phys. 0204, 013 (2002), arXiv:hep-th/0202021.

24. T. Ishii, G. Ishiki, S. Shimasaki and A. Tsuchiya, Phys. Rev. D 78, 106001 (2008), arXiv:0807.2352 [hep-th].

25. S. Catterall, E. Dzienkowski, J. Giedt, A. Joseph and R. Wells, J. High Energy Phys. 1104, 074 (2011), arXiv:1102.1725 [hep-th].

26. M. Hanada, J. High Energy Phys. 1011, 112 (2010), arXiv:1009.0901 [hep-lat].

27. R. Dijkgraaf and G. W. Moore, Commun. Math. Phys. 185, 411 (1997), arXiv:hepth/9608169.

28. F. Sugino, J. High Energy Phys. 0401, 015 (2004), arXiv:hep-lat/0311021.

29. R. C. Myers, J. High Energy Phys. 9912, 022 (1999), arXiv:hep-th/9910053. 
30. K. Sugiyama and K. Yoshida, Nucl. Phys. B 644, 128 (2002), arXiv:hep-th/0208029; S. R. Das, J. Michelson and A. D. Shapere, Phys. Rev. D 70, 026004 (2004), arXiv:hepth/0306270.

31. J. Kato, Y. Kondo and A. Miyake, J. High Energy Phys. 1109, 019 (2011), arXiv:1104.1252 [hep-th].

32. I. Kanamori, J. High Energy Phys. 1207, 021 (2012), arXiv:1202.2101 [hep-lat].

33. S. Iso, Y. Kimura, K. Tanaka and K. Wakatsuki, Nucl. Phys. B 604, 121 (2001), arXiv:hep-th/0101102.

34. H. Aoki, N. Ishibashi, S. Iso, H. Kawai, Y. Kitazawa and T. Tada, Nucl. Phys. B 565, 176 (2000), arXiv:hep-th/9908141.

35. A. Gonzalez-Arroyo and M. Okawa, Phys. Rev. D 27 (1983) 2397; T. Eguchi and R. Nakayama, Phys. Lett. B 122, 59 (1983).

36. S. Minwalla, M. Van Raamsdonk and N. Seiberg, J. High Energy Phys. 0002, 020 (2000), arXiv:hep-th/9912072.

37. S. Mandelstam, Nucl. Phys. B 213, 149 (1983); L. Brink, O. Lindgren and B. E. W. Nilsson, Phys. Lett. B 123, 323 (1983).

38. J. K. Erickson, G. W. Semenoff and K. Zarembo, Nucl. Phys. B 582, 155 (2000), arXiv:hep-th/0003055.

39. A. Matusis, L. Susskind and N. Toumbas, J. High Energy Phys. 0012, 002 (2000), arXiv:hep-th/0002075.

40. A. Hashimoto and N. Itzhaki, Phys. Lett. B 465, 142 (1999), arXiv:hep-th/9907166; J. M. Maldacena and J. G. Russo, J. High Energy Phys. 9909, 025 (1999), arXiv:hepth/9908134.

41. M. Hanada, S. Matsuura and F. Sugino, Nucl. Phys. B 857, 335 (2012), arXiv:1109.6807 [hep-lat]. 